\title{
Мощные непрерывные лазеры InGaAs/AIGaAs (1070 нм) с расширенным латеральным волноводом мезаполосковой конструкции
}

\author{
(C) И.С. Шашкин, А.Ю. Лешко, В.В. Шамахов, Н.В. Воронкова, В.А. Капитонов, К.В. Бахвалов, \\ С.О. Слипченко, Н.А. Пихтин, П.С. Копьев
}

Физико-технический институт им. А.Ф. Иоффе Российской академии наук, 194021 Санкт-Петербург, Россия

E-mail: shashkin@mail.ioffe.ru

Поступила в Редакцию 1 декабря 2020 г.

В окончательной редакции 10 декабря 2020 г.

Принята к публикации 10 декабря 2020 г.

Были разработаны полупроводниковые лазеры с латеральным волноводом мезаполосковой конструкции шириной 10 мкм и исследованы их излучательные характеристики. Показано, что непрерывная оптическая мощность для лазеров с длиной резонатора 4.6 мм достигает $2.6 \mathrm{~B}$ т при температуре теплоотвода $25^{\circ} \mathrm{C}$. Лазеры с меньшей длиной резонатора ( $<3$ мм) демонстрировали меньшее значение максимальной оптической мощности, что было связано с тепловым насыщением ватт-амперной характеристики.

Ключевые слова: полупроводниковый лазер, излучательные характеристики, оптическая мощность, тепловое насыщение.

DOI: 10.21883/FTP.2021.04.50736.9565

\section{1. Введение}

Задача увеличения выходной мощности и яркости непрерывного лазерного излучения важна для ряда практических приложений: прямая обработка материалов, накачка оптических волокон и др. Типично полупроводниковые лазеры с широкой излучающей апертурой $(W \sim 100$ мкм) обладают непрерывной выходной мощностью > 10 Вт $[1,2]$, однако многомодовая структура излучения существенно снижает яркость. Общий подход основан на использовании полупроводниковых лазеров с узким мезаполосковым волноводом, обеспечивающим одномодовый режим генерации. Однако ширина излучающей апертуры является ограничивающим фактором для получения высокой выходной оптической мощности. Поэтому поиск решений, позволяющих поднять выходную мощность без катастрофического падения качества выходного излучения, является актуальной задачей. Один из подходов основан на расширении выходной апертуры, что обеспечит повышение выходной мощности при условии сохранения одномодового или маломодового режима генерации.

В работе [3] исследованы лазеры, излучающие на длине волны $980 \mathrm{Hм}$, с узким мезаполоском и гетероструктурой на основе одномерного фотонного кристалла, позволяющей получить расходимость в перпендикулярной плоскостию. Исследования показали, что расширение полоска до 7 мкм позволяет повысить максимальную выходную мощность до 2 Вт, которая ограничена катастрофической оптической деградацией зеркал резонатора. 95\% мощности находится в угле 14 и $38^{\circ}$ для медленной и быстрой оси соответственно. Расширение полоска до 10 мкм привело к многомодовому режиму генерации и повышению параметра M2 с 1.5 до 3.5, при этом отсутствует информация о том, позволил ли данный шаг увеличить максимальную мощность или нет.

В работе [4] представлены экспериментальные исследования ватт-амперных характеристик в непрерывном режиме генерации для полупроводниковых лазеров с узким мезаполосковым контактом, изготовленных на основе гетероструктуры с двойной асимметрией и расширенным волноводом (an extreme double asymmetric (EDAS)). В работе исследовались полупроводниковые лазеры с шириной полоска от 5 до 15 мкм. Авторам удалось продемонстрировать максимальную оптическую мощность 2.7 Вт для лазеров с шириной полоска 15 мкм и параметром М2 $=2.2$, при этом в $8.5^{\circ}$ заключено 95\% энергии для мощности 1 Вт.

Ранее нами были исследованы одномодовые лазеры с узким мезаполосковым контактом ( $W \sim 5$ мкм), изготовленные на основе гетероструктур различных дизайнов $[5,6]$. Было показано, что использование сверхузких волноводов позволяет снизить расходимость в перпендикулярной плоскости до $18.5^{\circ}$, при этом максимальная мощность в непрерывном режиме достигала 550 мВт и была ограничена перегревом активной области [5]. Существенно улучшить мощностные характеристики одномодовых лазеров удалось за счет использования асимметричной гетероструктуры с широким волноводом [6]. Максимальная мощность в непрерывном режиме достигала 1600 мВт при ширине мезаполоска 5.5 мкм [6].

В рамках данной работы представлены результаты исследований непрерывных мощностных характеристик полупроводниковых лазеров с расширенным волноводом мезаполосковой конструкции для решения задачи повышения выходной оптической мощности. 
Расчетные значения модального усиления для различных мод при ширине латерального волновода мезаполосковой конструкции 5,10 и 20 мкм

\begin{tabular}{c|l|c|c|c|c|c}
\hline \multirow{2}{*}{$\begin{array}{c}\text { Ширина } \\
\text { полоска, мкм }\end{array}$} & \multicolumn{5}{|c}{ Модальное усиление, см $^{-1}$} \\
\cline { 2 - 7 } & TEM $_{00}$ & TEM $_{10}$ & TEM $_{20}$ & TEM $_{30}$ & TEM $_{40}$ & TEM $_{50}$ \\
\hline 5 & 15.30 & 5.4 & - & - & - & - \\
10 & 16.32 & 16.34 & 16.27 & - & - & - \\
20 & 16.332 & 16.337 & 16.339 & 16.336 & 16.258 & 15.043
\end{tabular}

\section{2. Экспериментальные образцы}

Для экспериментальных исследований использовалась асимметричная гетероструктура с широким волноводом, выращенная методом МОС-гидридной эпитаксии. Структура включала эмиттеры $p$ - и $n$-типов проводимости на основе $\mathrm{Al}_{x} \mathrm{Ga}_{1-x} \mathrm{As}(x=0.3)$, волноводный слой на основе $\mathrm{Al}_{x} \mathrm{Ga}_{1-x} \mathrm{As}(x=0.1)$ толщиной 1.7 мкм, активную область на основе одной квантовой ямы InGaAs толщиной 9 нм, смещенную относительно центра волноводного слоя в сторону $p$-эмиттера на 0.2 мкм. На основе данной структуры изготавливались полупроводниковые лазеры мезаполосковой конструкции.

На первом этапе была проведена оценка характеристик расширенного мезаполоскового волновода. В таблице показаны значения модального усиления для мод различных порядков, полученные при оптимальной глубине остаточного слоя, которая определялась как расстояние от активной области до поверхности в области мезаканавки. Видно, что максимальной селективностью обладает конструкция с шириной мезаполоска 5 мкм. Для данной конструкции максимальным усилением обладает 0 мода, а моды высших порядков имеют значительные потери. Увеличение ширины мезаполоска до 10 мкм приводит к тому, что разница модальных усилений между 0, 1 и 2 модами в оптимальной конструкции мезаполоскового волновода снижается до $0.08 \mathrm{~cm}^{-1}$, что не позволяет осуществить эффективную селекцию и обеспечить одномодовый режим генерации. При этом селективность относительно других мод высшего порядка остается существенной. Дальнейшее расширение мезаполоскового волновода до 20 мкм дает существенное снижение селективности для мод высших порядков и пороговые условия могут быть выполнены для первых шести мод, что может заметно снизить характеристики направленности и яркости.

Для решения задачи повышения оптической мощности без существенных потерь в яркости на основании сделанных оценок были изготовлены экспериментальные образцы полупроводниковых лазеров с мезаполосковым волноводом шириной 10 мкм. Экспериментальные образцы изготавливались двух длин резонаторов: 3 и 4.6 мм. Для всех образцов на грани резонатора наносились просветляющие $(5 \%)$ и отражающие $(95 \%)$ покрытия. Для экспериментальных исследований образцы напаивались $p$-стороной вниз на медные теплоотводы с помощью индиевого припоя.

\section{3. Результаты эксперимента и их обсуждение}

В первой части экспериментальных исследований проводились измерения ватт-амперных характеристик (BтAX) в непрерывном режиме генерации. В процессе измерений поддерживалась постоянная температура теплоотвода $25^{\circ} \mathrm{C}$. Для лазерных кристаллов с длиной резонатора 3 мм максимальная выходная оптическая мощность достигала $2.4 \mathrm{BT}$ при токе 4 А и ограничивалась насыщением ВтAX (рис. 1). Стоит отметить, что непрерывная ВТАХ для лазеров с длиной резонатора 3 мм и шириной полоска 5.5 мкм насыщалась на токе 2.35 А при мощности $1.7 \mathrm{BT}$ [6], тогда как лазеры, исследуемые в данной работе, при сопоставимых токах и мощностях имели ВтАХ, близкую к линейной. Оценка теплового режима работы была проведена на основании спектров лазерной генерации (рис. 2,a). Для полученных спектров был сделан расчет перегрева активной области из смещения спектра в длинноволновую область. Для расчетов перегрева использовался коэффициент $0.35 \mathrm{Hм} /{ }^{\circ} \mathrm{C}$. Видно, что на токе $2.5 \mathrm{~A}$ перегрев достигает $41^{\circ} \mathrm{C}$. Также стоит отметить особенность начального участка, ВтАХ, которая выражена в сверхлинейном росте мощности. Данный эффект можно связать с влиянием перегрева активной области на характеристики латерального волновода. Известно, что увеличение температуры приводит к росту показателя преломления. В результате локального разогрева в области мезаполоскового контакта имеет место усиление латерального волновода, что приводит к усилению локализации моды в области полоскового кон-

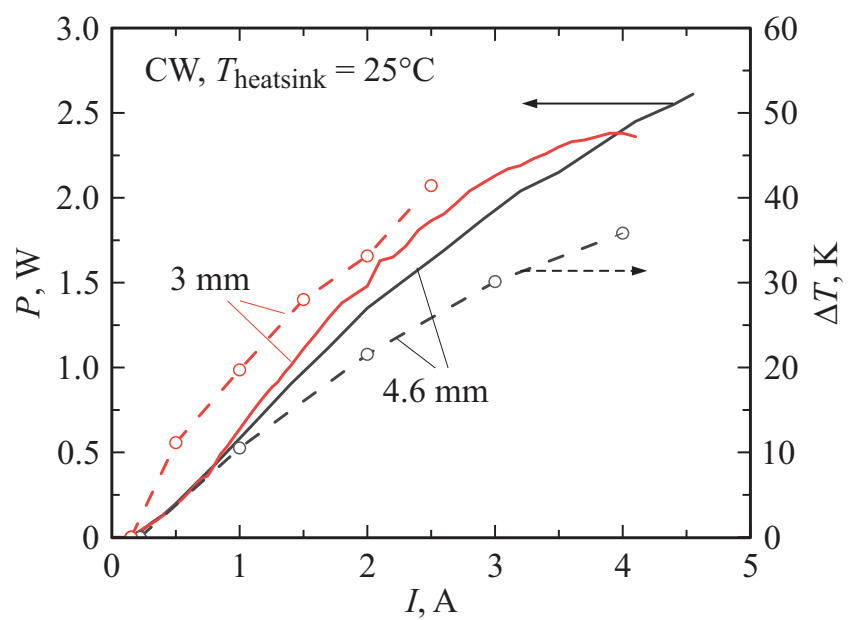

Рис. 1. Зависимость непрерывной оптической мощности от тока накачки для лазеров с длиной резонатора 4.6 и 3 мм (непрерывные линии) и температуры активной области от тока накачки (пунктир, пустые круги). Температура теплоотвода $25^{\circ} \mathrm{C}$. 


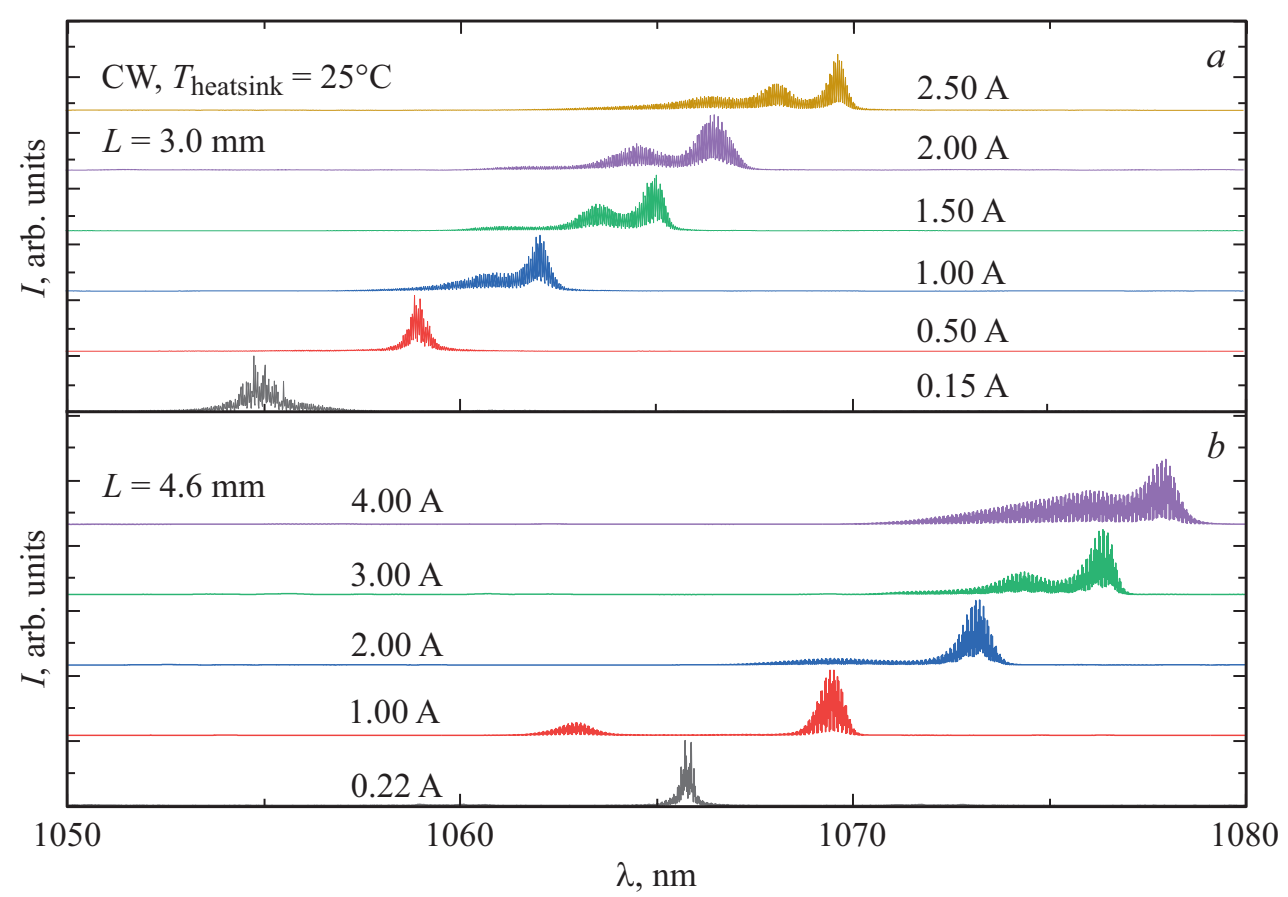

Pис. 2. Спектры генерации в непрерывном режиме в зависимости от тока накачки для лазеров с длиной резонатора 3 мм $(a)$ и 4.6 мм (b). Температура теплоотвода $25^{\circ} \mathrm{C}$.

такта, где усиление максимально, и снижению доли моды в области мезаканавок, где оптические потери выше.

С целью ослабления эффекта перегрева активной области в области высоких токов накачки были исследованы кристаллы с длиной резонатора 4.6 мм. Лазеры с длиной резонатора 4.6 мм имели меньшее значение средней начальной дифференциальной эффективности. Однако за счет низких внутренних оптических потерь в гетероструктуре $\left(\sim 0.6 \mathrm{~cm}^{-1}\right)$ данная разница заметно проявлялась только для токов $>2 \mathrm{~A}$. В результате максимальная разность выходной оптической мощности между лазерами с длиной резонатора 3 и 4.6 мм достигалась на токе 3 А и составила $200 \mathrm{мBт} \mathrm{(рис.} \mathrm{1).} \mathrm{Для} \mathrm{больших} \mathrm{значений}$ тока ВтАХ лазеров с длиной резонатора 3 мм испытывала насыщение, тогда как ВтАХ лазеров с длиной резонатора 4.6 мм сохраняла зависимость, близкую к линейной. Основная причина такого поведения связана со снижением теплового сопротивления в области максимальных токов на $\sim 40 \%$ для лазеров с длиной резонатора 4.6 мм, что обеспечило более эффективный отвод тепла. $\mathrm{B}$ результате характер $\mathrm{BTAX}$, близкий к линейному, сохранялся вплоть до тока $4.5 \mathrm{~A}$, что позволило получить максимальную мощность 2.6 Вт, при этом признаки теплового насыщения ВтАХ отсутствовали. Исследования спектров генерации образцов с длиной резонатора 4.6 мм (рис. 2,b) показали, что максимальный перегрев составил $35^{\circ} \mathrm{C}$, при этом в исследованном диапазоне токов накачки отсутствовали признаки сверхлинейного роста температуры. Исследования ВтАХ показали, что на зависимости присутствуют области нелинейности, Такой характер типичен для одномодовых лазеров и связан с проявлением таких эффектов, как включение новых мод или перестроение модовых структур, а также увод луча („,beam steering“'). Данные эффекты меняют расходимость излучения в дальней зоне в плоскости, параллельной слоям гетероструктуры, так как именно изменение свойств латерального волновода определяет особенности, описанные выше. Действительно, измеренная расходимость поля в перпендикулярной плоскости, определяемая дизайном гетероструктуры, составила $29^{\circ}$ на уровне 1/2 от максимума и не менялась во всем диапазоне токов накачки. Для плоскости, параллельной слоям гетероструктуры, изменения тока сопровождались перестроениями в параллельном поле. Наиболее характерные формы параллельного поля, соответствующие различным значениям тока накачки, приведены на рис. 3 . Видно, что около порога генерации параллельное поле включает один лепесток. Экспериментальное значение расходимости на уровне $1 / 2$ от максимума составило $\sim 2.6^{\circ}$, что меньше расчетной величины для нулевой моды $4.6^{\circ}$ (см. рис. 3). Данный эффект может объясняться тем, что около порога генерации в экспериментальных образцах латеральный волновод слабее расчетного случая. Как отмечалось выше, на ослабление волновода может оказывать влияние избыточная концентрация носителей заряда, накопленная в области инжекции, под полосковым контактом. С ростом тока накачки за счет термического разогрева области инжекции происходит усиление латерального волновода, что сопровождается включением мод высшего порядка и увеличением расходимости параллельного поля (рис. 3). При этом общий профиль определяется суперпозицией 0,1 и 2 мод, а мак- 


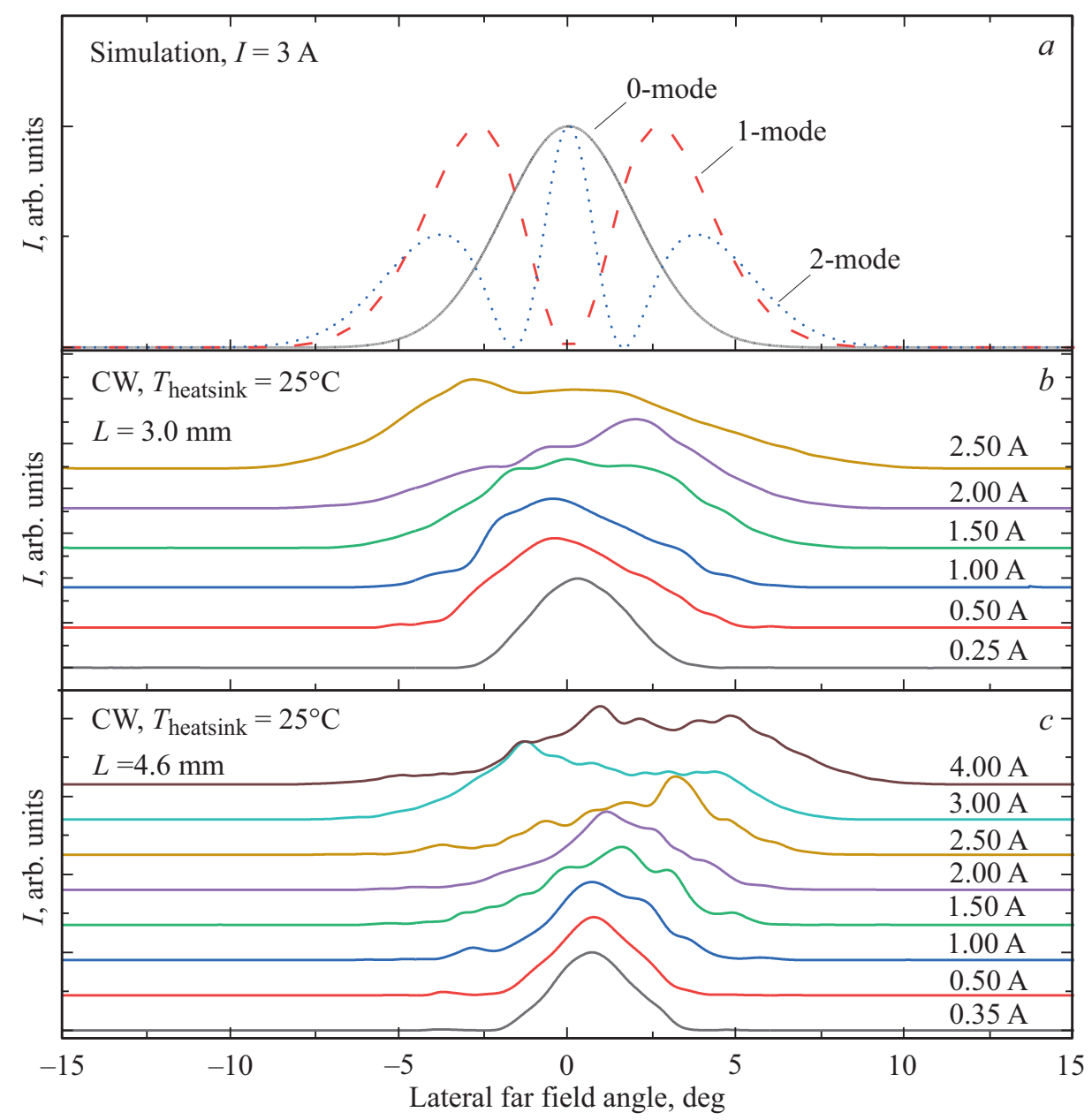

Рис. 3. Расходимость излучения в дальней зоне, в плоскости, параллельной слоям гетероструктуры, полученная при расчете для 0,1 и 2 мод латерального волновода $(a)$, и в экспериментальных измерениях при различных значениях непрерывного тока накачки для лазеров с длиной резонатора 3 мм $(b)$ и 4.6 мм $(c)$. Температура теплоотвода $25^{\circ} \mathrm{C}$.

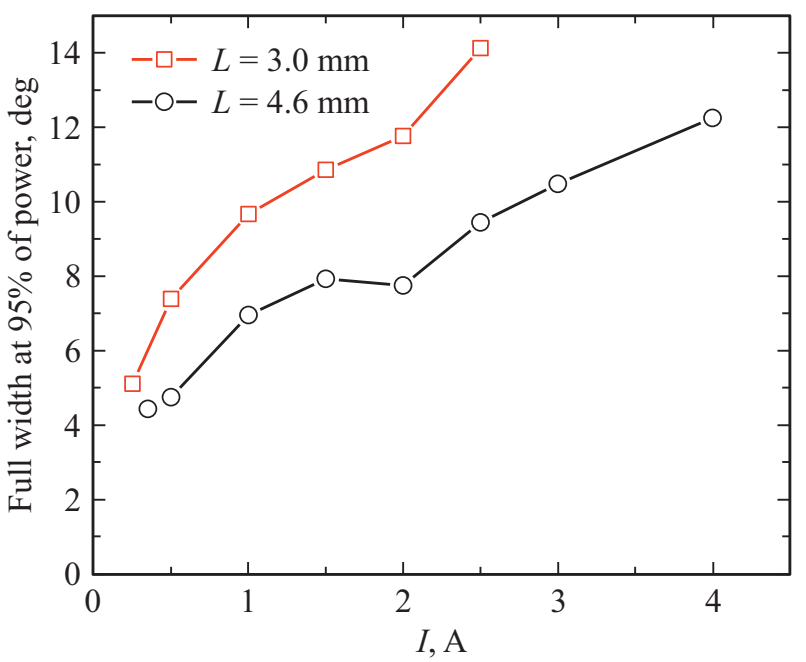

Рис. 4. Значения расходимости излучения в дальней зоне, в плоскости, параллельной слоям гетероструктуры, заключающие 95\% мощности, полученные для различных значений токов накачки для лазеров с длиной резонатора 3 мм (красные квадраты) и 4.6 мм (черные круги). Температура теплоотвода $25^{\circ} \mathrm{C}$. симальная ширина определяется модой второго порядка. На рис. 4 показана зависимость ширины дальнего поля, включающей 95\% излучаемой мощности. Видно, что при одинаковых значениях тока накачки расходимость излучения лазера с длиной резонатора 3 мм всегда выше, чем лазера с длиной резонатора 4.6 мм. Вклад в данный эффект может давать больший локальный разогрев области инжекции лазеров с меньшей длиной резонатора (рис. 1). Расчет, сделанный на основании полученных результатов, показал, что латеральная яркость на токе $4 \mathrm{~A}$ для образца $L=4.6$ мм составляет $3.7 \mathrm{BT} /($ мм $\cdot$ мрад), что в $\sim 2$ раза больше по сравнению с $1.75 \mathrm{BT} /($ мм - мрад) в работе [3] и сопоставимо с результатом, достигнутым в работе [4] (4.3 Вт/(мм - мрад)). В работе [7] проведено моделирование и показано, что по мере расширения полоска и появления латеральных мод более высокого порядка латеральная яркость будет увеличиваться и составит 2 Вт/(мм · мрад) для апертуры 23 мкм. Нами же экспериментально подтверждено увеличение яркости при расширении апертуры до 10 мкм и достигнут более оптимистичный результат. 


\section{4. Заключение}

Проведенные исследования показали, что использование расширенных латеральных волноводов мезаполосковой конструкции, оптимизированных для работы в маломодовом режиме, позволяет существенно увеличить непрерывную выходную оптическую мощность без заметного ухудшения качества лазерного излучения. Полученные результаты стали возможны за счет увеличения длины лазерных кристаллов без заметного падения дифференциальной эффективности, что было обеспечено использованием асимметричной гетероструктуры с низкими оптическими потерями.

\section{Финансирование работы}

Исследования выполнены при финансовой поддержке гранта Российского научного фонда (проект № 19-79-30072).

\section{Конфликт интересов}

Авторы заявляют, что у них нет конфликта интересов.

\section{Список литературы}

[1] С.О. Слипченко, Д.А. Винокуров, Н.А. Пихтин, З.Н. Соколова, А.Л. Станкевич, И.С. Тарасов, Ж.И. Алфёров. ФТП, 38 (12), 1477 (2004).

[2] K.H. Hasler, H. Wenzel, P. Crump, S. Knigge, A. Maasdorf, R. Platz, G. Erbert. Semicond. Sci. Technol., 29 (4), 045010 (2014).

[3] S. Zhao, Y. Wang, H. Qu, Y. Liu, X. Zhou, A. Liu, W. Zheng. IEEE Photon. Technol. Lett., 29 (23), 2005 (2017).

[4] M. Wilkens, H. Wenzel, J. Fricke, A. Maaßdorf, P. Ressel, S. Strohmaier, G. Tränkle. IEEE Photon. Technol. Lett., 30 (6), 545 (2018)

[5] И.С. Шашкин, А.Ю. Лешко, Д.Н. Николаев, В.В. Шамахов, Д.А. Веселов, Н.А. Рудова, П.С. Копьев. ФТП, 54 (4), 414 (2020).

[6] И.С. Шашкин, А.Ю. Лешко, Д.Н. Николаев, В.В. Шамахов, Н.А. Рудова, К.В. Бахвалов, Н.А. Пихтин. ФТП, 54 (4), 408 (2020).

[7] J.-P. Koester, A. Putz, H. Wenzel, H.-J. Wünsche, M. Radziunas, H. Stephan, M. Wilkens, A. Zeghuzi, A. Knigge. Semicond. Sci. Technol., 36, 015014 (2021).

Редактор Г.А. Оганесян

\section{High-power CW InGaAs/AIGaAs (1070 nm) lasers with a broadened lateral waveguide of a mesa-stripe structure}

\author{
I.S. Shashkin, A.Yu. Leshko, V.V. Shamakhov, \\ N.V. Voronkova, V.A. Kapitonov, K.V. Bakhvalov, \\ S.O. Slipchenko, N.A. Pikhtin, P.S. Kop'ev \\ loffe Institute, \\ 194021 St. Petersburg, Russia
}

\begin{abstract}
Semiconductor lasers with a $10 \mu \mathrm{m}$ wide lateral waveguide of a mesa-stripe design were developed and their light characteristics were studied. Lasers with a $4.6 \mathrm{~mm}$ long cavity is shown to have a continuous wave $(\mathrm{CW})$ optical power of $2.6 \mathrm{~W}$ at a heatsink temperature of $25^{\circ} \mathrm{C}$. Lasers with a shorter cavity $(<3 \mathrm{~mm})$ showed a lower value of the maximum optical power due to a thermal rollover of the light-current curve.
\end{abstract}

